\title{
Kinematic and electromyographic assessment of manual handling on a supermarket green- grocery shelf
}

\author{
Alessio Silvetti ${ }^{\mathrm{a}}$, Silvia Mari ${ }^{\mathrm{b}}$, Alberto Ranavolo ${ }^{\mathrm{a}}$, Federico Forzano ${ }^{\mathrm{a}}$, Sergio Iavicoli ${ }^{\mathrm{a}}$, \\ Carmela Conte ${ }^{\mathrm{c}}$ and Francesco Draicchio ${ }^{\mathrm{a}, *}$ \\ ${ }^{\mathrm{a}}$ INAIL - Department of Occupational Medicine, Rome, Italy \\ ${ }^{\mathrm{b}}$ Fondazione Don Gnocchi, Milan, Italy \\ ${ }^{\mathrm{c}}$ Department of Neuroscience, University of Pavia, Pavia, Italy
}

Received 10 January 2013

Accepted 8 January 2014

\begin{abstract}
.
BACKGROUND: There are few epidemiological data regarding musculoskeletal disorders (MSDs) in retail industry. Biomechanical risk assessment in ergonomics is commonly performed in retail sector using standardized protocols. However, such protocols have numerous limitations, such as the lack of objectivity or applicability and restrictive conditions.

OBJECTIVE: The aim of this study was to analyze one of the most commonly used shelves in vegetable and fruit departments in order to investigate the effect of different shelf levels (i.e. with variations in height and horizontal distance) and load weights on the workers' biomechanical load.

METHODS: We investigated trunk, shoulder, elbow, hip, knee and ankle joint ROMs, as well as the mean and peak EMG values of the upper limb, trunk and lower limb muscles.

RESULTS: We found that shelf level has a significant effect on most of the parameters examined, whereas within this limited range of 6 and $8 \mathrm{~kg}$, weight does not affect the biomechanical load. We also identified the shelf levels that place the least and most strain on the musculoskeletal system.

CONCLUSIONS: We therefore recommend that the height and horizontal distance be carefully considered when shelves are being designed. Kinematic and EMG approach may help to objectively assess shelf-related risks. Our findings are in agreement with RNLE LI values and therefore support RNLE.
\end{abstract}

Keywords: Retail sector, ergonomics, movement analysis, shelf levels, NIOSH protocol

\section{Introduction}

The retail industry includes a wide range of types of companies, which differ in size, goods sold, worker activities, structure and management. The developed countries in which this industry is growing [14] include

*Corresponding author: Francesco Draicchio, INAIL - Department of Occupational Medicine, Via Fontana Candida 1, Monte Porzio Catone, Rome, 00040, Italy. Tel.: +39 0694181373; Fax: +39 0694181410; E-mail: f.draicchio@inail.it.
Italy, as demonstrated by the Italian workers' compensation authority (INAIL. http://bancadati.inail.it/preve nzionale/). However, there are few epidemiological data regarding musculoskeletal disorders (MSDs) in workers in the retail industry, possibly owing to the fact that there is a high turnover in employees, who tend to be young and rarely gain more than a few years of experience.

When Gardner et al. [12] analyzed back accidents involving thousands of retail merchandise store workers whose jobs consisted in manual material handling 
$(\mathrm{MMH})$, they found that the accident rate was highest among workers with a heavy workload and limited work experience.

An interesting study carried out on Italian supermarkets and hypermarkets [27] showed a 12-month storewide low back pain (LBP) prevalence of $35 \%$, and revealed that the fruit and vegetable departments were those with the highest biomechanical risk. High lifting frequencies, heavy weights, prolonged and marked trunk inclination, which are all required in such departments, are generally accepted as the main causes of musculoskeletal injuries.

Forcier et al. reported a high prevalence of workrelated MSDs in supermarket workers in a study they conducted [11]. They also demonstrated that the Canadian workers' compensation authority data on MSDs are underestimated if compared with those yielded by the Nordic questionnaire [19], thus highlighting the need for further biomechanical investigations in supermarket workers.

Biomechanical risk assessment in ergonomics in the retail industry is usually performed using standardized protocols, which however have numerous limitations [26].

For example, the PATH protocol [2], which has been adopted to assess the risks of MMH in retail workers who perform a range of non-repetitive work activities [22], is not quantitative.

Similarly, Coyle [4] assessed the risks of MMH in supermarkets by adopting two standardized protocols, i.e. the REBA [17] and the New Zealand Code of Practice for Manual Handling Hazard Control Record [9], to gain a better understanding of the work environment. However, the former is based on subjective evaluations and the latter is a questionnaire whose score is based on categorized measurements of joint posture.

By contrast, the National Institute for Occupational Safety and Health (NIOSH) protocol [28], which has been widely adopted because it provides a quantitative biomechanical assessment of risk $[13,20,23,25$, 30], cannot always be applied owing to its lifting task limitations and restrictions. Indeed, it has been found that half of the jobs have a recommended weight limit (RWL) of zero, which is attributed to the fact that the parameters measured exceeded the bounds of the RNLE [18]. For instance, if the horizontal distance, which is required for a correct assessment of moment and biomechanical stress [6], exceeds $63 \mathrm{~cm}$, the RWL is equal to zero.

Despite the growing amount of attention being paid to retail workers, quantitative and objective meth- ods, such as surface electromyography (sEMG) and stereophotogrammetry, have seldom been used to measure the biomechanical overload caused by muscle effort and kinematics.

The biomechanical load related to MMH activities in the retail industry is associated with both the goods weight and the shelf level, which determines the heights and the horizontal distances at the beginning and at the end of the lifting action. However, shelf level is partially determined by marketing needs dictated by the visibility and reachability of the goods.

To our knowledge, no study has yet investigated the quantitative differences in biomechanical load during manual handling in supermarket shelves. Thus, the aim of this study was to analyze, from a kinematic and electromyographic perspective, one of the most common shelves used in vegetable and fruit departments in order to assess the effect of different shelf levels and load weights on the workers' biomechanical load. Our findings may help to design a shelf that combines workers' safety and marketing needs.

\section{Materials and methods}

\subsection{Participants}

Five male workers with more than 3 years of working experience were enrolled in the study; the workers' mean (SD) age, height and weight were 31 years (3.3), $173 \mathrm{~cm} \mathrm{(3.2)} \mathrm{and} 74 \mathrm{~kg}(6.5)$, respectively, and their elbow height was $108.2 \mathrm{~cm}(1.8)$. The workers voluntarily performed the study trials in the laboratory. None of the participants had a history of either musculoskeletal disorders or neurological diseases, or had recently taken any drugs. All the participants gave their written consent after they had received a full explanation of the study procedure. The study was approved by the local ethics committee and conformed to the Helsinki declarations. No information regarding expected results was provided in order to avoid the results being biased, whether consciously or unconsciously.

\subsection{Instrumentation}

An optoelectronic motion analysis system (SMARTE System, BTS, Milan, Italy) [10], consisting of eight infra-red ray cameras (operating at $120 \mathrm{~Hz}$ ), was used. Anthropometric data were collected for each subject, and spherical markers, covered with aluminum powder reflecting material, were placed over prominent 
bony landmarks using simplified versions of the Rab model [24] for the upper limbs, and of the Davis model [8] for the trunk and lower limbs. Twenty markers were placed as follows: one on the cutaneous projections of the spinous processes of the $7^{\text {th }}$ cervical vertebra, one over the sacrum, while the remainder were placed bilaterally over the acromion, the oleocranon, the ulnae, the anterior superior iliac spine, the great trochanter, the lateral femoral condyle, the lateral malleolus and the fifth metatarsal head. Four markers were also placed at the edge of the crate being handled. The markers were attached in such a way that they could not fall out of place during data acquisition.

A calibration procedure was performed before the first data were captured. Kinematic data were acquired and digitized with a sampling rate of $120 \mathrm{~Hz}$. Spatial accuracy was $<0.2 \mathrm{~mm}$ in the calibrated volume, which was approximately $4 \mathrm{~m}$ long, $2 \mathrm{~m}$ wide and $2.30 \mathrm{~m}$ high.

Electrical muscle activity was recorded using a 16 channel Wi-Fi surface electromyography system (FreeEMG, BTS SpA, Milan, Italy) at a sampling frequency of $1 \mathrm{kHz}$. A pre-processing filtering and noise removal procedure was performed. The lower and upper cut-off frequencies of the Hamming filter were 10 $\mathrm{Hz}$ and $400 \mathrm{~Hz}$, respectively, while the common mode reaction ratio was $100 \mathrm{~dB}$.

After skin preparation, surface electromyographic signals were detected from each muscle by two Ag/ $\mathrm{AgCl}$ pre-gelled disposable surface electrodes (H124 SG, Kendall ARBO, Donau, Germany) which had a detection surface of $10 \mathrm{~mm}$ (gelled). Electrodes were placed in the direction of the muscle fibers, according to the European Recommendations for Surface Electromyography (SENIAM) [16], with a centre-to-centre distance of $20 \mathrm{~mm}$. We investigated the following muscles on the right side of the body: deltoideus anterior (DA), biceps brachii (BB), latissimus dorsi (LD), erector spinae (ES) and rectus femoris (RF).

In order to elicit the maximal voluntary isometric contraction (MVCi) from each muscle, six isometric exertions were performed, according to SENIAM indications [16].

Data acquisition from the optoelectronic cameras and the sEMG signal were synchronized and integrated.

\subsection{Experimental procedures}

The shelf used in the greengrocery section was installed in the laboratory. The shelf had four levels on

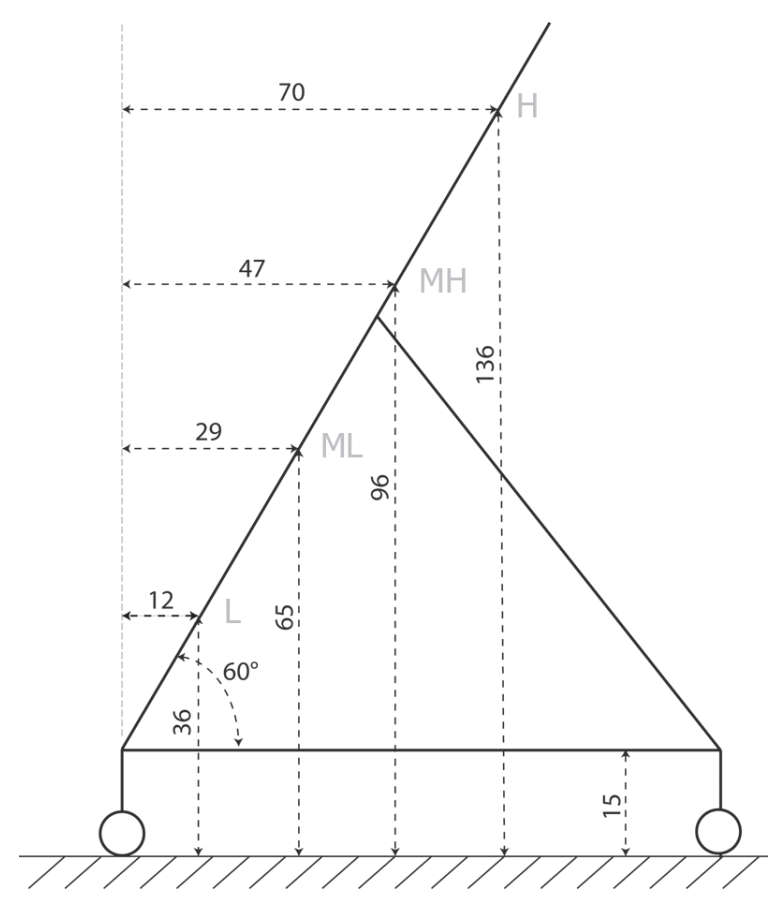

Fig. 1. Schematic representation of the four different heights and horizontal distances of the shelf $(\mathrm{cm})$.

which the crate could be positioned: high $(\mathrm{H})$, middle high (MH), middle low (ML) and low (L) as summarized in Table 1 and illustrated in Fig. 1. Before starting formal measurements, which took place in a quiet room with normal indoor temperature and lighting, the subjects performed a practice session to familiarize themselves with the experimental procedure and were instructed on how to execute the task correctly (with some pauses to avoid fatigue). During the experimental sessions, the subjects lifted the crate 10 times onto each of the four levels ( 5 times with $6 \mathrm{~kg}$ and the other 5 with $8 \mathrm{~kg}$ ), thus totaling 40 lifts. The order of the 6 and $8 \mathrm{~kg}$ lifts was randomly assigned. Loads of 6 and $8 \mathrm{~kg}$ were chosen because an investigation had shown that they were the weights most commonly shifted in the department. Workers were asked to raise a crate (height: $15 \mathrm{~cm}$; width: $40 \mathrm{~cm}$; depth: $30 \mathrm{~cm}$ ) with side handles $20 \mathrm{~cm}$ above the bottom. In the starting position, subjects stood facing the shelf with the elbow flexed at $90^{\circ}$. As regards the horizontal starting position, participants were positioned in such a way that their malleolus lay $5 \mathrm{~cm}$ from the vertical projection of the anterior edge of the lowest shelf. Before they started, the crate was supported by an adjustable tool, at the subject's elbow level. They then placed the load on one of the four levels of the shelf. A five-minute rest was allowed between groups of five trials in order to avoid muscle 
Table 1

Height and horizontal distance of each of the four shelf levels and their LIs

\begin{tabular}{lrcr}
\hline Shelf levels & Height & Horizontal distance & LI 6 Kg \\
\hline High (H) & $136 \mathrm{~cm}$ & $70 \mathrm{~cm}$ & - \\
Midde High (MH) & $96 \mathrm{~cm}$ & $47 \mathrm{~cm}$ & 0.55 \\
Middle Low (ML) & $65 \mathrm{~cm}$ & $29 \mathrm{~cm}$ & 0.73 \\
Low (L) & $36 \mathrm{~cm}$ & $12 \mathrm{~cm}$ & 0.45 \\
\hline
\end{tabular}

Table 2

The first part of the table shows the effect of weight, level and the weight-level interaction ( $p$ values) and the $\mathrm{R}^{2}$ values of the general linear model analysis of the kinematic parameters. The ANOVA results for the two weights are shown in the last two columns of the table. Bold values indicate $p<0.05$

\begin{tabular}{lcccccc}
\hline & Weight & Level & Weight ${ }^{*}$ level & $\mathrm{R}^{2}$ & Anova $6 \mathrm{~kg}$ & Anova $8 \mathrm{~kg}$ \\
\hline ROM-S & 0.501 & $\mathbf{0 . 0 0 0}$ & 0.138 & 0.968 & $\mathbf{0 . 0 0 0}$ & $\mathbf{0 . 0 0 0}$ \\
ROM-E & 0.065 & $\mathbf{0 . 0 0 0}$ & 0.901 & 0.933 & 0.988 & $\mathbf{0 . 0 0 1}$ \\
ROM-T & 0.951 & $\mathbf{0 . 0 0 0}$ & 0.418 & 0.966 & $\mathbf{0 . 0 0 0}$ \\
ROM-Hi & 0.172 & $\mathbf{0 . 0 0 0}$ & $\mathbf{0 . 0 3 6}$ & 0.837 & $\mathbf{0 . 0 0 0}$ \\
ROM-K & $\mathbf{0 . 0 2 5}$ & $\mathbf{0 . 0 0 0}$ & 0.259 & 0.888 & $\mathbf{0 . 0 2 1}$ \\
ROM-A & 0.241 & $\mathbf{0 . 0 0 0}$ & 0.841 & $\mathbf{0 . 0 0 0}$ & $\mathbf{0 . 0 0 0}$ \\
\hline
\end{tabular}

fatigue. Table 1 reports the Revised NIOSH Lifting Indexes (LI) for the four levels and both weights [28,29]. We didn't apply the Revised NIOSH Lifting Equation (RNLE) to $\mathrm{H}$ level because of the horizontal restriction (horizontal distance exceeding $63 \mathrm{~cm}$ ).

\subsection{Data processing}

The data yielded by a frame-by-frame tracking procedure (Smart Tracker, BTS, Milan, Italy) were processed using Analyzer software (Smart Analyzer, BTS, Milan, Italy).

The $\mathrm{x}$-axis of the local Cartesian coordinate system lay on the subject's sagittal plane. The beginning and end of the task were defined, respectively, as the instants in which the right lower marker on the crate started and stopped moving along the $\mathrm{x}$-axis. After $5 \mathrm{~Hz}$ low-pass filtering, shoulder (S), elbow (E), trunk (T), hip (Hi), knee (K) and ankle (A) joint angular displacement in the sagittal plane were determined. The task duration was reduced to 100 samples using a polynomial procedure. For each angle, the range of motion (ROM) was calculated as the difference between the maximum and minimum values during the task.

The sEMG signals were rectified, integrated with a mobile window of $0.125 \mathrm{~s}$, filtered with a $5 \mathrm{~Hz}$ Hamming low-pass filter and normalized to the maximum value of the MVCi. The mean activation and the peak values of the task were then calculated.

\subsection{Statistical analysis}

All the analyses were performed using SPSS Statistics 17.0 software (SPSS Inc., Chicago, IL, USA). The mean and standard deviations (SD) of all the kinematic and electromyographic parameters were calculated for each weight and shelf level. The Shapiro-Wilk test was applied to verify the null hypothesis that each parameter of the acquired sample came from a normally distributed population.

A general linear model analysis was performed to investigate the effect of weight and of shelf level (main effects), as well as of their interaction, on the parameters. A one-way repeated-measures analysis of variance (ANOVA) was also performed for each weight to determine whether there was a significant effect of shelf level on the mean values of the parameters. A parametric paired t-test with Bonferroni's correction between pairs of shelf level was applied separately for the two weights investigated. A parametric paired t-test was used to detect any significant differences between $6 \mathrm{~kg}$ and $8 \mathrm{~kg}$ for each shelf level. $P$-values of less than 0.05 were considered statistically significant.

\section{Results}

The Shapiro-Wilk test showed that all the variables considered were normally distributed.

\subsection{Kinematic results}

The results of the general linear model analysis and of the ANOVA are shown in Table 2. A significant effect of level was found in all the joint ROMs, whereas the weight had a significant effect only on knee ROM and the weight-level interaction had a significant effect only on hip joint ROM. ANOVA revealed a signif- 
Table 3

Comparison of kinematic parameters between pairs of shelf levels. Bold values indicate $p<0.0083$ at post-hoc test after Bonferroni's correction

\begin{tabular}{|c|c|c|c|c|c|c|c|c|c|c|c|c|}
\hline & \multicolumn{2}{|c|}{ L Vs ML } & \multicolumn{2}{|c|}{ L Vs MH } & \multicolumn{2}{|c|}{ L Vs H } & \multicolumn{2}{|c|}{ ML Vs MH } & \multicolumn{2}{|c|}{ ML Vs H } & \multicolumn{2}{|c|}{$\mathrm{MH} \mathrm{Vs} \mathrm{H}$} \\
\hline & $6 \mathrm{~kg}$ & $8 \mathrm{~kg}$ & $6 \mathrm{~kg}$ & $8 \mathrm{~kg}$ & $6 \mathrm{~kg}$ & $8 \mathrm{~kg}$ & $6 \mathrm{~kg}$ & $8 \mathrm{~kg}$ & $6 \mathrm{~kg}$ & $8 \mathrm{~kg}$ & $6 \mathrm{~kg}$ & $8 \mathrm{~kg}$ \\
\hline ROM-S & 0.021 & 0.000 & 1 & 0.129 & 0.000 & 0.000 & 0.008 & 0.000 & 0.000 & 0.000 & 0.000 & 0.000 \\
\hline ROM-E & 1 & 0.012 & 0.066 & 0.000 & 0.002 & 0.000 & 0.022 & 0.000 & 0.001 & 0.000 & 0.166 & 0.000 \\
\hline ROM-T & 0.000 & 0.000 & 0.000 & 0.000 & 0.000 & 0.000 & 0.744 & 1 & 0.001 & 0.000 & 0.004 & 0.000 \\
\hline ROM-Hi & 0.000 & 0.000 & 0.000 & 0.000 & 0.000 & 0.000 & 1 & 1 & 0.023 & 0.000 & 0.053 & 0.000 \\
\hline ROM-K & 0.221 & 0.059 & 1 & 0.250 & 1 & 0.009 & 1 & 1 & 0.026 & 0.000 & 0.153 & 0.001 \\
\hline ROM-A & 0.000 & 0.003 & 0.000 & 0.019 & 0.013 & 0.595 & 1 & 0.969 & 0.001 & 0.030 & 0.004 & 0.305 \\
\hline
\end{tabular}

Shoulder ROM
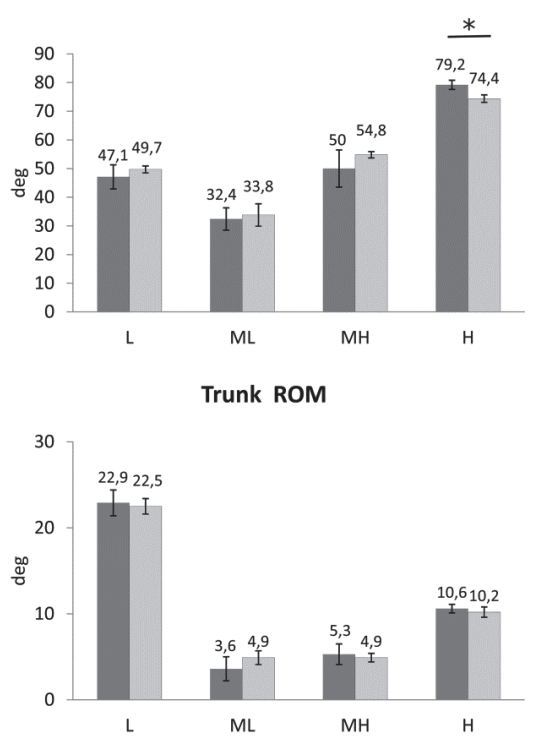

Knee ROM

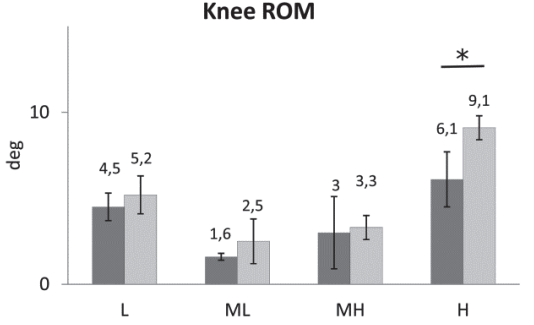

Elbow ROM
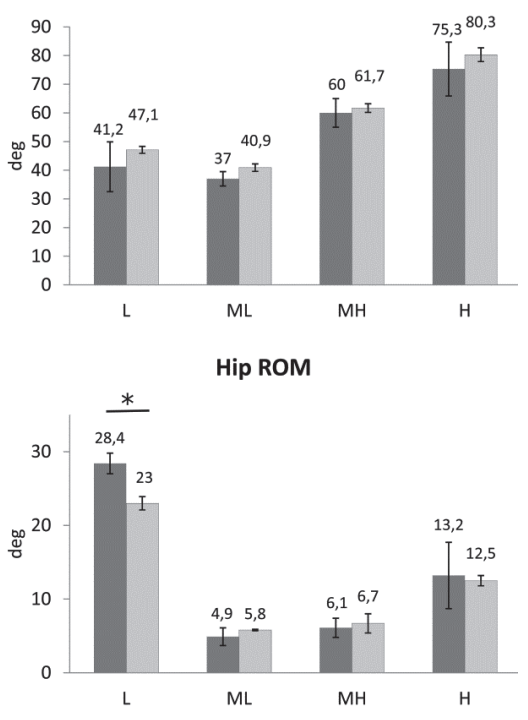

Ankle ROM

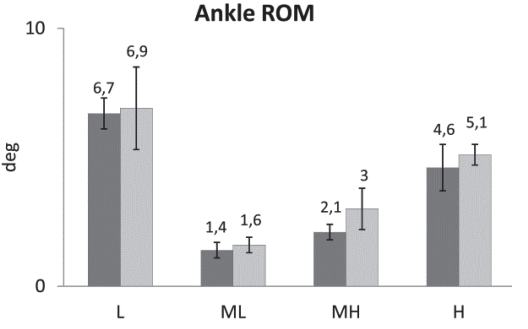

Fig. 2. Comparison between the two weights (dark gray $=6 \mathrm{~kg}$; light gray $=8 \mathrm{~kg}$ ) in the kinematic parameters for each shelf level. ${ }^{*} p<0.05$ at Student t-test.

icant effect of shelf level on all the joint ROM for both weights.

Figure 2 shows the between-weight comparisons in the joint ROMs for each shelf level. A significant difference was observed only in level $\mathrm{H}$ for knee ROM (lower with $6 \mathrm{~kg}$ ). Figures 3 and 4 show the mean angle curves of the shoulder, elbow, trunk, hip, knee and ankle for the four shelf levels for the 6 and $8 \mathrm{~kg}$ crates, respectively.

The results of the between-level comparisons are shown in Table 3. Some comparisons were significant for some ROM. A particularly high number of significant differences were found between the following pairs of levels for both weights: ML vs. H, MH vs. H and $\mathrm{L}$ vs. $\mathrm{H}$ (9, 8 and 8 significant differences, respectively).

\subsection{Electromyographic results}

\subsubsection{Mean EMG values}

The results of the general linear model analysis and of the ANOVA are shown in Table 4. A significant 
Table 4

The first part of the table shows the effect of weight, level and the weight-level interaction ( $\mathrm{p}$ values) and the $\mathrm{R}^{2}$ values of the general linear model analysis of the mean EMG values. The ANOVA results for the two weight are shown in the last two columns of the table. Bold values indicate $p<0.05$

\begin{tabular}{lcccccc}
\hline & Weight & Level & Weight ${ }^{*}$ level & $\mathrm{R}^{2}$ & Anova $6 \mathrm{~kg}$ & Anova $8 \mathrm{~kg}$ \\
\hline DA & $\mathbf{0 . 0 0 3}$ & $\mathbf{0 . 0 0 0}$ & 0.104 & 0.974 & $\mathbf{0 . 0 0 0}$ & $\mathbf{0 . 0 0 0}$ \\
BB & $\mathbf{0 . 0 0 0}$ & $\mathbf{0 . 0 0 0}$ & 0.003 & 0.950 & $\mathbf{0 . 0 0 1}$ \\
LD & 0.099 & $\mathbf{0 . 0 0 0}$ & 0.452 & 0.980 & $\mathbf{0 . 0 0 0}$ \\
ES & 0.001 & $\mathbf{0 . 0 0 0}$ & 0.052 & 0.875 & $\mathbf{0 . 0 0 4}$ \\
RF & 0.156 & 0.001 & 0.479 & 0.687 & $\mathbf{0 . 0 2 0}$ \\
\hline
\end{tabular}
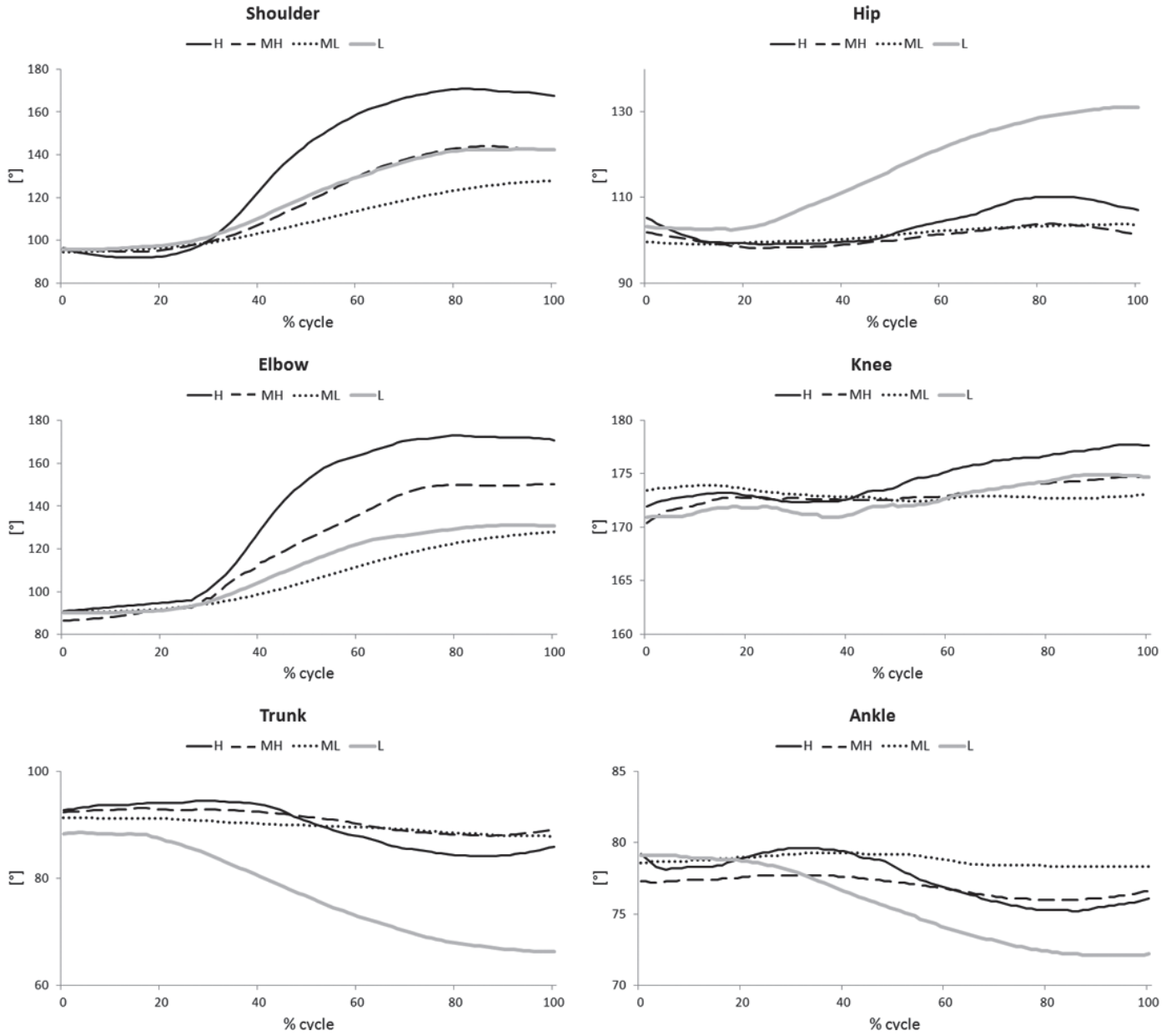

Fig. 3. Mean angle curves of shoulder, elbow, trunk, hip, knee and ankle for the four shelf levels used for the $6 \mathrm{~kg}$ crate.

effect of level was found in all the mean EMG values. Weight had a significant effect on the mean EMG value of $\mathrm{DA}, \mathrm{BB}$ and $\mathrm{ES}$, whereas the weight-level interaction only had a significant effect on the mean EMG value of BB. ANOVA revealed a significant effect of shelf level on all the mean EMG values for both weights, except for RF with $8 \mathrm{~kg}$.

Figure 6 shows the between-weight comparisons in the mean EMG values for each shelf level. A signif- icant difference was observed in the BB mean EMG values in the ML, $\mathrm{MH}$ and $\mathrm{H}$ levels, with values being lower with $6 \mathrm{~kg}$. Furthermore, the ES mean EMG value in the $\mathrm{H}$ level proved to be significantly lower with 6 $\mathrm{kg}$ than with $8 \mathrm{~kg}$.

The results of the between-level comparisons are shown in Table 5. Some comparisons were statistically significant for some muscles. A particularly high number of significant differences emerged between the fol- 
Table 5

Comparison of mean EMG values between pairs of shelf level. Bold values indicate $p<0.0083$ at post-hoc test after Bonferroni's correction

\begin{tabular}{|c|c|c|c|c|c|c|c|c|c|c|c|c|}
\hline & \multicolumn{2}{|c|}{ L Vs ML } & \multicolumn{2}{|c|}{ L Vs MH } & \multicolumn{2}{|c|}{ L Vs H } & \multicolumn{2}{|c|}{ ML Vs MH } & \multicolumn{2}{|c|}{ ML Vs H } & \multicolumn{2}{|c|}{ MH Vs H } \\
\hline & $6 \mathrm{~kg}$ & $8 \mathrm{~kg}$ & $6 \mathrm{~kg}$ & $8 \mathrm{~kg}$ & $6 \mathrm{~kg}$ & $8 \mathrm{~kg}$ & $6 \mathrm{~kg}$ & $8 \mathrm{~kg}$ & $6 \mathrm{~kg}$ & $8 \mathrm{~kg}$ & $6 \mathrm{~kg}$ & $8 \mathrm{~kg}$ \\
\hline DA & 1 & 1 & 0.001 & 0.164 & 0.000 & 0.000 & 0.000 & 0.168 & 0.000 & 0.000 & 0.000 & 0.000 \\
\hline BB & 0.064 & 0.010 & 0.008 & 0.000 & 0.001 & 0.000 & 1 & 0.010 & 0.037 & 0.000 & 0.350 & 0.003 \\
\hline LD & 1 & 1 & 0.048 & 0.035 & 0.000 & 0.000 & 0.033 & 0.006 & 0.000 & 0.000 & 0.000 & 0.000 \\
\hline ES & 1 & 1 & 1 & 1 & 0.014 & 0.002 & 0.555 & 0.377 & 0.005 & 0.001 & 0.071 & 0.010 \\
\hline $\mathrm{RF}$ & 1 & 1 & 0.439 & 0.099 & 0.139 & 0.670 & 0.116 & 0.061 & 0.039 & 0.408 & 1 & 1 \\
\hline
\end{tabular}
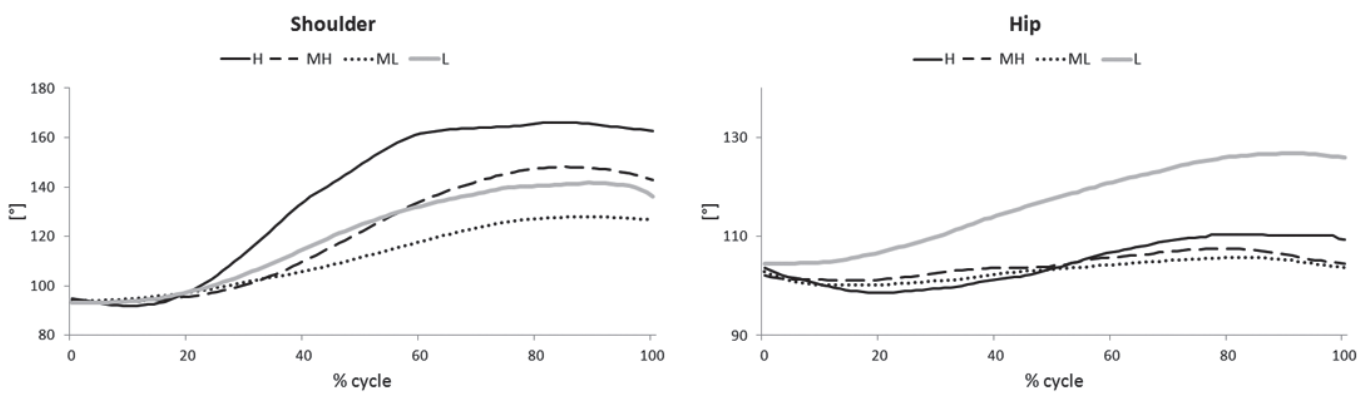

Elbow
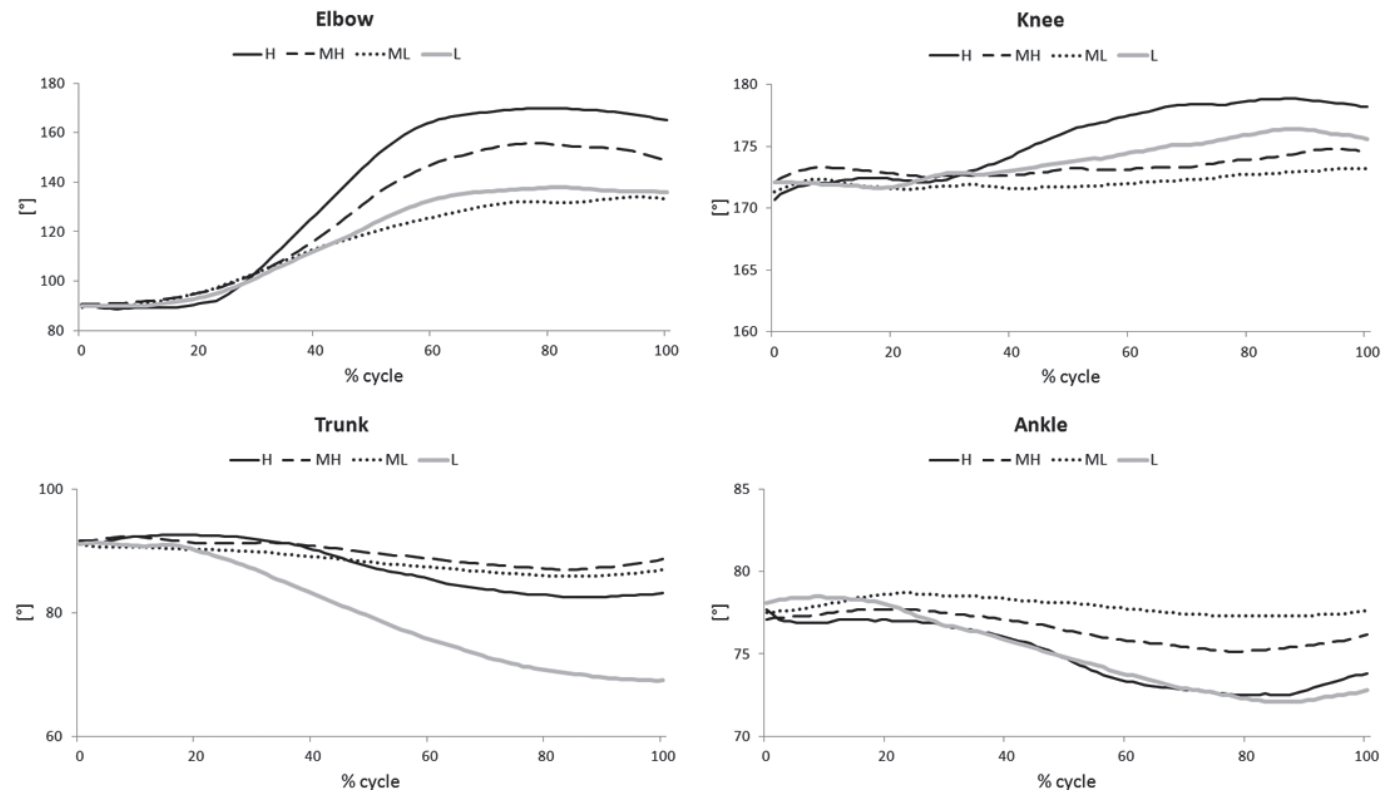

Fig. 4. Mean angle curves of shoulder, elbow, trunk, hip, knee and ankle for the four shelf levels for the $8 \mathrm{~kg}$ crate.

lowing pairs of level for both weights: ML vs. H, MH vs. $H$ and L vs. H (7, 6 and 6 significances, respectively).

\subsubsection{Peak EMG values}

The results of the general linear model analysis and of the ANOVA are shown in Table 6. A significant effect of level was found in all the peak EMG values, except for RF. Weight had a significant effect on the peak EMG value of BB and RF. No significant effect was found for the weight-level interaction on the peak EMG value. ANOVA revealed a significant effect of shelf level on all the peak EMG values for both weights, except for RF, which was not significant for either weight.

Figure 6 shows the between-weight comparisons in the peak EMG values for each shelf level. A significant difference was only observed in the DA peak EMG values in the L level, with values being lower with $6 \mathrm{~kg}$.

The results of the between-level comparisons are shown in Table 7. All the comparisons were statistically significant, except for the pairs L vs. ML and ML 
Table 6

The first part of the table shows the effect of weight, level and the weight-level interaction ( $\mathrm{p}$ values) and the $\mathrm{R}^{2}$ values of the general linear model analysis of the peak EMG values. The ANOVA results for the two weight are shown in the last two columns of the table. Bold values indicate $p<0.05$

\begin{tabular}{lcccccc}
\hline & Weight & Level & Weight * level & $\mathrm{R}^{2}$ & Anova $6 \mathrm{~kg}$ & Anova $8 \mathrm{~kg}$ \\
\hline DA & 0.406 & $\mathbf{0 . 0 0 0}$ & 0.873 & 0.974 & $\mathbf{0 . 0 0 0}$ & $\mathbf{0 . 0 0 0}$ \\
BB & $\mathbf{0 . 0 0 4}$ & $\mathbf{0 . 0 0 0}$ & 0.340 & 0.933 & $\mathbf{0 . 0 0 0}$ \\
LD & 0.958 & $\mathbf{0 . 0 0 0}$ & 0.506 & 0.966 & $\mathbf{0 . 0 0 0}$ \\
ES & 0.080 & $\mathbf{0 . 0 0 0}$ & 0.445 & 0.825 & $\mathbf{0 . 0 0 0}$ \\
RF & $\mathbf{0 . 0 3 4}$ & 0.489 & 0.994 & 0.333 & 0.384 \\
\hline
\end{tabular}
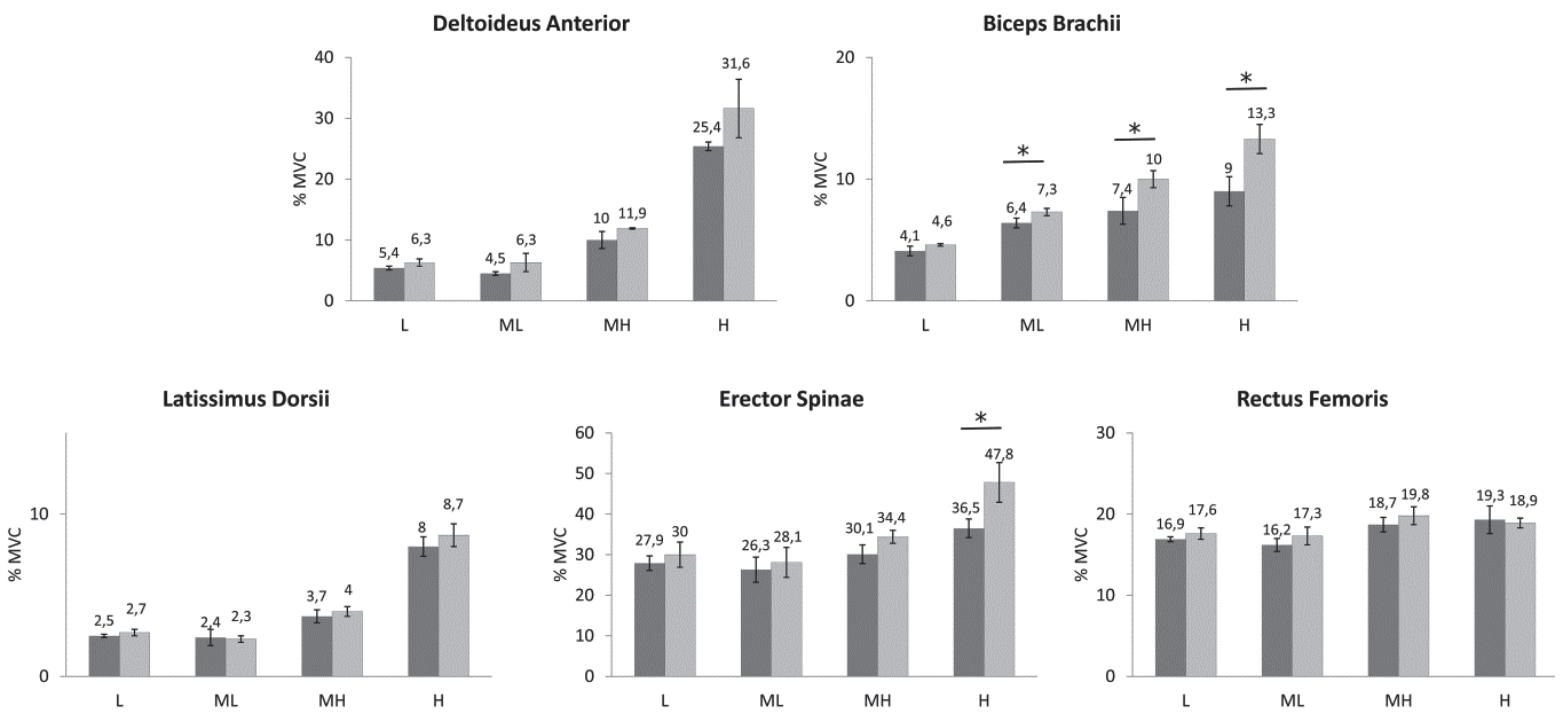

Fig. 5. Comparison between the two weights (dark gray $=6 \mathrm{~kg}$; light gray $=8 \mathrm{~kg}$ ) in the mean EMG values for each shelf level. ${ }^{*} p<0.05$ at Student t-test.
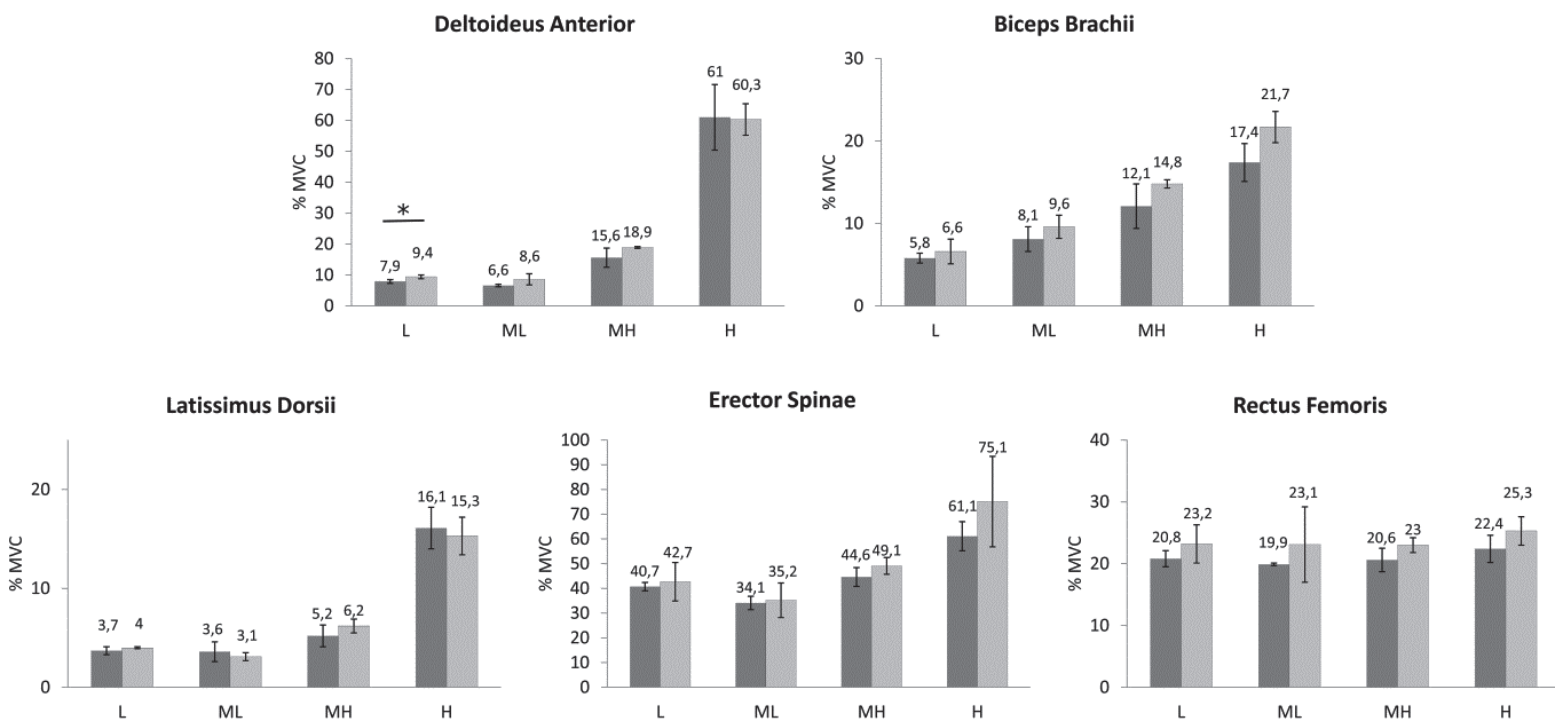

Fig. 6. Comparison between the two weights (dark gray $=6 \mathrm{~kg}$; light gray $=8 \mathrm{~kg}$ ) in the peak EMG values for each shelf level. ${ }^{*} p<0.05$ at Student t-test. 
Table 7

Comparison of peak EMG values between pairs of shelf levels. Bold values indicate $p<0.0083$ at post-hoc test after Bonferroni's correction

\begin{tabular}{|c|c|c|c|c|c|c|c|c|c|c|c|c|}
\hline & \multicolumn{2}{|c|}{ L Vs ML } & \multicolumn{2}{|c|}{ L Vs MH } & \multicolumn{2}{|c|}{$\mathrm{L} \mathrm{Vs} \mathrm{H}$} & \multicolumn{2}{|c|}{ ML Vs MH } & \multicolumn{2}{|c|}{ ML Vs H } & \multicolumn{2}{|c|}{$\mathrm{MH} \mathrm{Vs} \mathrm{H}$} \\
\hline & $6 \mathrm{~kg}$ & $8 \mathrm{~kg}$ & $6 \mathrm{~kg}$ & $8 \mathrm{~kg}$ & $6 \mathrm{~kg}$ & $8 \mathrm{~kg}$ & $6 \mathrm{~kg}$ & $8 \mathrm{~kg}$ & $6 \mathrm{~kg}$ & $8 \mathrm{~kg}$ & $6 \mathrm{~kg}$ & $8 \mathrm{~kg}$ \\
\hline$\overline{\mathrm{DA}}$ & 1 & 1 & 0.761 & 0.016 & 0.000 & 0.000 & 0.478 & 0.010 & 0.000 & 0.000 & 0.000 & 0.000 \\
\hline BB & 1 & 0.179 & 0.028 & 0.001 & 0.001 & 0.000 & 0.227 & 0.013 & 0.002 & 0.000 & 0.059 & 0.002 \\
\hline LD & 1 & 1 & 1 & 0.181 & 0.000 & 0.000 & 1 & 0.036 & 0.000 & 0.000 & 0.000 & 0.000 \\
\hline ES & 0.416 & 1 & 1 & 1 & 0.001 & 0.035 & 0.062 & 0.618 & 0.000 & 0.012 & 0.005 & 0.100 \\
\hline RF & 1 & 1 & 1 & 1 & 1 & 1 & 1 & 1 & 0.669 & 1 & 1 & 1 \\
\hline
\end{tabular}

vs MH. A particularly high number of significant differences emerged between the following pairs of levels for both weights: ML vs. H, L vs. H and MH vs. H (7, 7 and 6 significances, respectively).

\section{Discussion}

The aim of this study was to evaluate the biomechanical effort made by experienced workers while lifting crates of different weights onto supermarket shelves positioned at different heights. We selected employees with more than three years of experience in order to prevent the confounding factor of inexperience, reported by Authier et al. [1] and Chen et al. [3], from influencing our results.

The weights we investigated were those most commonly handled in the greengrocery section of the supermarket. Although heavier crates $(14 \mathrm{~kg}$ and $18 \mathrm{~kg}$ ) were shifted in the department, they were not considered in this study because these crates were lifted in the supermarket selling area by means of a mechanical or electric pallet jack. The movement we analyzed was free of any trunk torsion because the asymmetry factor, which was extremely low in our experimental setup, is only relevant when torsion values exceed $60^{\circ}$ [5] (condition not observed in our preliminary inspections). Although the frequency of the movement is clearly relevant to the overall risk of injury, this study was not designed to address this issue.

The main finding that emerges from our study is that shelf level exerted a far greater influence on task performance than the difference between 6 and $8 \mathrm{~kg}$ weights, as previously reported also by Habes et al. [15] and Olivera et al. [21]. Our results are in keeping with those obtained by Das [5], who reported that the height of the shelf plays a crucial role in determining the muscle effort required during load handling. The negligible influence of weight on the kinematic and EMG parameters may be due to the relatively small difference between the two loads investigated. Indeed, Davis and Marras [7] also reported that small differences in weight have little effect on spinal load.

In the present study, we found that the ML shelf level yielded lower mean and peak muscular activity values than the $\mathrm{L}, \mathrm{MH}, \mathrm{H}$ shelf levels as well as the lowest joint ROM values. The biggest differences emerged between the ML and $\mathrm{H}$ levels. These results suggest that ML is the level that induces the least biomechanical load, muscular effort and joint ROM.

Our findings are in agreement with RNLE LI values (Table 1). In fact ML horizontal and vertical distances are close to RNLE optimal values. Therefore our experimental data support RNLE.

Our data indicate that the $\mathrm{H}$ and $\mathrm{L}$ levels induce the greatest biomechanical exertion and ROM, with the former requiring marked shoulder flexion and the latter significant trunk flexion. Indeed, the $\mathrm{H}$ level, which has a horizontal distance of $70 \mathrm{~cm}$, not only yielded high elbow extension and shoulder flexion values on the sagittal plane, but also marked trunk and upper limb muscle activation. The results we obtained for the $\mathrm{H}$ level are particularly worthy of note because the NIOSH protocol does not assess a horizontal distance greater than $63 \mathrm{~cm}$. The L level instead yielded, owing to its low vertical distance, higher trunk and hip flexion and ankle dorsiflexion ROM values. However, the L level also yielded the lowest mean and peak BB activity values, which are due to the contribution made by gravity and by "hanging-down arm posture" during the lowering movement.

\section{Conclusion}

To prevent back injuries and upper limb disorders among greengrocery supermarket workers, we analyzed the role of shelf height and horizontal distance. Our data show that shelf level plays a role in biomechanical load by influencing muscle effort and joint ROMs. In particular, we identified the shelf levels that exert the least and the most strain on the musculoskeletal system. The least stressful shelf proved to be the 
ML. We therefore recommend that the height and horizontal distance be carefully considered when shelves are being designed. Shelves could be designed in such a way as to bring them closer to the worker in order to reduce the horizontal distance.

Stores in which space is limited may optimize shelf height and distance according to the results of our study, avoiding, if possible, horizontal displays.

Moreover, we believe that the kinematic and EMG approach may help to objectively assess shelf-related risks, according to standardized protocols, such as NIOSH and REBA. Indeed, a careful evaluation of the working environment design may help to reduce the costs of working days lost as a result of occupational diseases as well as of the expense that may be incurred to redesign an unhealthy work environment.

\section{References}

[1] Authier M, Lortie M, Gagnon M. Manual handling techniques: comparing novices and experts. Int. J. Ind. Ergon. 1996; 17(5): 419-429.

[2] Buchholz B, Paquet V, Punnett L, Lee D, Moir S. PATH: A work sampling-based approach to ergonomic job analysis for construction and other non-repetitive work. Appl. Ergon. 1996; 27(3): 177-187.

[3] Chen YL, Lee YC, Chen CJ. Differences in lifting strength profiles between experienced workers and novices at various exertion heights. Int. J. Ind. Ergon. 2011; 41(1): 53-58.

[4] Coyle A. Comparison of the Rapid Entire Body Assessment and the New Zealand Manual Handling 'Hazard Control Record' for assessment of manual handling hazards in the supermarket industry. Work. 2005; 24: 111-116.

[5] Das B, Mabaleka G. An electromyographic investigation of asymmetric lifting and moving of a load: I. effects of load lift from floor to target shelves. Hum. Fact. and Ergon. in Manufact. 2009 ; 19(3): 254-274.

[6] Dampsey PG, Burdorf A, Fathallah FA, Sorock GS, Hashemi L. Influence of measurement accuracy on the application of the 1991 NIOSH equation. Appl. Ergon. 2001; 32(1): 91-99.

[7] Davis KG, Marras WS. Assessment of the relationship between box weight and trunk kinematics: does a reduction in box weight necessarily correspond to a decrease in spinal loading?. Hum. Fact. 2000; 42(2): 195-208.

[8] Davis RB, Ounpuu S, Tyburski D, Gage JR. A gait analysis data collection and reduction technique. Hum. Movement Sci. 1991; 10: 575-587.

[9] Department of Labour Te Tar Mahi. Code of practice for manual handling [internet]. Wellington (New Zealand): Occupational Safety and Health service of the Department of Labour, the Accident Compensation Corporation; 2001; [updated 2012 May 24; cited 2011 Oct 17]. Available from: http:// www.osh.dol.govt.nz/order/catalogue/pdf/manualcode.pdf.

[10] Ferrigno G, Pedotti A. ELITE: a digital dedicated hardware system for movement analysis via real-time TV signal processing. IEEE T. Bio-Med. Eng. 1985; 32(11): 943-950.

[11] Forcier L, Lapointe C, Lortie M, Buckle P, Kuorinka I, Lemaire J, Beaugrand S. Supermarket workers: their work and their health, particularly their self-reported musculoskeletal problems and compensable injuries. Work. 2000; 30: 493510.

[12] Gardner LI, Landsittel DP, Nelson NA. Risk factors for back injury in 31,076 retail merchandise store workers. Am. J. Epidemiol. 1999; 150(8): 825-833.

[13] Gentzler M, Stader S. Posture stress on firefighters and emergency medical technicians (EMTs) associated with repetitive reaching, bending, lifting, and pulling tasks. Work. 2010: 37(3); 227-239.

[14] Guay M. L'industrie du commerce recrute en permanence. Le Guide de l'emploi et des entreprises qui recrutent. Edition 2004-2005, Septembre Éditeur, 2004.

[15] Habes D, Carlson W. Budger D. Muscle fatigue associated with repetitive arm lifts: effects of height, weight and reach. Ergonomics. 1985; 28(2): 471-488.

[16] Hermes, H., Freriks, B., Merletti, R. et al. European recommendations for surface ElectroMyoGraphy (SENIAM). Ed CLUT 2000.

[17] Hignett S, McAtamney L. Rapid Entire Body Assessment (REBA). Appl. Ergon. 2000; 31(2): 201-205.

[18] Karwoski W, and Brokaw N. Implications of the proposed revisions in a draft of the revised NIOSH lifting guide (1991) for job redesign: A field study. Proceedings of the 36th Annual Meeting of the Human Factors Society. 1992; 36(10): 659-663.

[19] Kuorinka I, Jonsson B, Kilborn A, Vinterberg H, BieringSorensen F, Andersson G, Jorgensen K. Standardised Nordic questionnaires for the analysis of musculoskeletal symptoms. Appl. Ergon. 1987; 18(3): 233-237.

[20] Okimoto MLLR, Teixeira ER. Proposed procedures for measuring the lifting task variables required by the Revised NIOSH Lifting Equation - A case study. Int. J. Ind. Ergonom. 2009; 39(1): 15-22.

[21] Oliveira AB, Silva LCCB, Coury HJCG How do low/high height and weight variation affect upper limb movements during manual material handling of industrial boxes? Rev. Bras. Fisioter. 2011; 15(6): 494-502.

[22] Pan CS, Gardner LI, Landsittel DP, Hendricks SA, Chiou SS, Punnett L. Ergonomic exposure assessment: an application of the PATH systematic observation method to retail workers. Postures, Activities, Tools and Handling. Int. J. Occup. Environ. Hea. 1999; 5(2): 79-87.

[23] Plamondon A, Delisle A, Trimble K, Desjardins P, Rickwood T. Manual materials handling in mining: The effect of rod heights and foot positions when lifting "in-the-hole" drill rods. Appl. Ergon. 2006; 37(6): 709-718.

[24] Rab G, Petuskey Y, Bagley A. A method for determination of upper extremity kinematics. Gait Posture. 2002; 15(2): 113119.

[25] Resnick ML, Chaffin DB. An ergonomic evaluation of handle height and load in maximal and submaximal cart pushing. Appl. Ergon. 1995; 26(3): 173-178.

[26] Takala EP, Pehkonen I, Forsman M, Hansson GA, Mathiassen SE, Neumann WP, Sjøgaard G, Veiersted KB, Westgaard RH, Winkel J. Systematic evaluation of observational methods assessing biomechanical exposures at work. Scand. J. Work Env. Hea. 2010; 36(1): 3-24.

[27] Violante FS, Graziosi F, Bonfiglioli R, Curti S, Mattioli S. Relations between occupational, psychosocial and individual factors and three different categories of back disorder among supermarket workers. Int. Arch. Occ. Env. Hea. 2005; 78(8): 613-624.

[28] Waters TR, Putz-Anderson V, Garg A, Fine LJ. Revised 
NIOSH equation for the design and evaluation of manual lifting tasks. Ergonomics. 1993; 36(7): 749-776.

[29] Waters T, Putz-Anderson V, and Garg A. Applications Manual for the Revised Lifting Equation, (DHHS/NIOSH) Pub. No.
94-110. Cincinnati, Ohio: CDC, NIOSH, 1994.

[30] Wright EJ, Haslam RA. Manual handling risks and controls in a soft drinks distribution centre. Appl. Ergon. 1999; 30(4): 311-318. 